\title{
Letter about "The song of Anorexia Nervosa: a specific evoked potential response to musical stimuli in affected participants" by Spalatro et al. (https://doi.org/10.1007/s40519-020-00898-4), published online: 05 May 2020
}

\author{
Paolo Benna ${ }^{1}$ (1)
}

Received: 5 June 2020 / Accepted: 27 July 2020 / Published online: 6 August 2020

(c) Springer Nature Switzerland AG 2020

\begin{abstract}
In the article "The song of Anorexia Nervosa: a specific evoked potential response to musical stimuli in affected participants" by Spalatro et al., in the Methods section, a paragraph relating to N100 and P300 data acquisition is missing. Indeed, the stimulation paradigms and recording methods are not described. Furthermore, in the paragraph "N100 and P300 analysis", the related issues are missing. In the absence of the data mentioned, it is not possible to understand the results.
\end{abstract}

Dear Prof. Cuzzolaro,

I fear there was an inconvenience in the preparation of the material for the final version of the article "The song of Anorexia Nervosa: a specific evoked potential response to musical stimuli in affected participants" by Spalatro et al. (https://doi.org/10.1007/s40519-020-00898-4), published online: 05 May 2020.

In fact, in the Methods section, a paragraph relating to N100 and P300 data acquisition is missing, with the description of the stimulation paradigms and recording methods.

Furthermore, in the paragraph "N100 and P300 analysis", the related issues are not discussed, but the Authors only provide general information relating to the two event-related potentials. The data regarding the $\mathrm{N} 1$ consist in the proposing of some sentences present in the related Wikipedia entry
(https://en.wikipedia.org/wiki/Visual_N1), accompanied by an inappropriate bibliographic entry, because it relates to the Auditory N1 ([36] Tomé et al., 2015).

The material that is omitted is essential for understanding the results and possibly evaluating their reproducibility.

Yours sincerely,

Paolo Benna.

Publisher's Note Springer Nature remains neutral with regard to jurisdictional claims in published maps and institutional affiliations.
Paolo Benna

paolo.benna@unito.it

1 Neuroscience Department, Università di Torino, Turin, Italy 